Syrian refugee communities. The Syrian conflict has resulted in one of the greatest humanitarian crises of the $21^{\text {st }}$ century. Over 5.6 million Syrians have become refugees, mostly residing in the neighboring countries of Turkey, Jordan, and Lebanon. Bleak prospects for Syrian refugee youth have raised concerns of a 'lost generation' of children who have had their housing, schooling, and childhoods interrupted. The combination of inadequate national refugee policies, abject poverty, and withering international aid for the crisis have made child labor endemic among many Syrian refugee communities in Jordan, Lebanon, and Turkey (Küppers \& Ruhmann, 2016; International Labour Organization et al., 2017; Habib et al., 2019). This address will share stories that elucidate how child labor takes root in conflict settings and how the international community can better address the challenges facing those who are most vulnerable.

\section{K-05 INTERVENTION STUDIES IN OCCUPATIONAL HEALTH: THE MISSING LINK}

Martie van Tongeren. The University of Manchester, UK

\subsection{6/OEM-2019-EPI.5}

Interventions are very common in workplaces mainly to improve productivity and efficiencies, but also in order to comply with regulatory requirements (environmental and occupational) and to improve the health of workers. Consequently, exposure to chemical agents, at least in Western Europe and the US, have declined in the last 4-5 decades. However, there is a remarkable paucity of properly conducted intervention studies in occupational health, in particular in relation to chemical and biological agents and their health outcomes. As a result, we don't have a very good understanding of what interventions actually work to reduce exposure and to improve the health of workers. Very wide estimates of the effectiveness of control measures have been noted from observational studies, probably because only few studies were probably conducted and many studies just compared the exposure between workplaces with and without the intervention. However, there is an important role for properly conducted intervention studies to reduce exposure and improve occupational health, and results of properly designed intervention studies will lead to a better understanding of causality as well as better understanding of effective interventions.

\section{Pesticide Exposure Assessment}

\section{A.1 PESTICIDE EXPOSURE OF WORKERS DURING TREATMENT AND RE-ENTRY TASKS IN APPLE- GROWING: RESULTS FROM THE CANEPA STUDY IN FRANCE}

\footnotetext{
${ }^{1}$ Mathilde Bureau ${ }^{*},{ }^{1}$ Béatrix Béziat, ${ }^{2}$ Geoffroy Duporté, ${ }^{3}$ Yannick Lecluse, ${ }^{1}$ Xavier Schwall, ${ }^{3,4}$ Valérie Bouchart, ${ }^{3,4}{ }^{G}$ aëlle Riou, ${ }^{2}$ Emmanuelle Barron, ${ }^{2}$ Marie-Hélène Devier, ${ }^{5}$ Francis Macary, ${ }^{1}$ Alain Garrigou, ${ }^{2}$ Hélène Budzinski, ${ }^{3}$ Pierre Lebailly, ${ }^{1}$ Isabelle Baldi. ${ }^{1}$ Equipe EPICENE, Bordeaux Population Health Center, Inserm U1219, Université de Bordeaux, Bordeaux, France; ${ }^{2}$ Equipe LPTC, EPOC UMR 5805, Université de Bordeaux CNRS, Bordeaux, France; ${ }^{3} E q u i p e$ Anticipe, UMR 1086, Université de Caen-Normandie Inserm, Centre François Baclesse, Caen, France; ${ }^{4}$ Laboratoire LABEO, Caen, France; ${ }^{5}$ Equipe EABX, UR ETBX, IRSTEA, BordeauX - Cestas, France
}

10.1136/OEM-2019-EPI.6
Few studies were performed to assess workers' exposure to pesticides in orchards and even less during re-entry activities. However, nearly 40 pesticide treatments per year are carried out in apple-growing especially against fungal diseases, mainly scab. In order to characterize pesticide exposures of farmers in epidemiological studies, we performed a non-controlled field study in 3 regions of France (Normandy, South-West and Rhône-Alpes) in apple orchards during the 2016 and 2017 seasons. We observed the activity of the workers trying to disrupt it as little as possible. Workers' external contamination and their determinants were assessed during 158 working days, corresponding to 31 treatment days (including mixing, spraying and equipment cleaning), 69 re-entry days (including apple hand-thinning, anti-hail net opening and closing tasks) and 58 harvesting days. We performed both detailed observations of work characteristics on the whole day (including an ergonomic approach) and pesticide measurements for dermal contamination during each task (following the OCDE guidelines). Potential dermal exposure was measured with cotton pads placed onto the skin (11 body areas), and cotton gloves or hand rinsing for each task. Captan and dithianon, fungicides representative of pesticide use in apple-growing, were used as markers for exposure. Relative contribution of the different body areas to total exposure was assessed. In addition, to investigate potential sources of contamination, we measured dislodgeable residues in the environment of the workers by analyzing leaf and fruit samples and surfaces of work equipment. Results indicated that workers' dermal exposure was higher during re-entry tasks than treatments and harvests. The median dermal contamination during treatment was $4.03 \mathrm{mg}$ of active ingredients per day, levels ranging from $0.95 \mathrm{mg}$ to $64.19 \mathrm{mg}$. Most of the contamination was observed on the hands, especially during mixing. We will focus our presentation on levels and main determinants of pesticide exposure during treatment tasks.

\section{A.2 EXPOSURE TO BENZIMIDAZOLE FUNGICIDES IN AGRICULTURE AND NON-HODGKIN LYMPHOMAS, OVERALL AND BY SUBTYPES, IN THE AGRICULTURE AND CANCER (AGRICAN) COHORT}

\begin{abstract}
${ }^{1,2}$ Amandine Busson*, ${ }^{1,3}$ Séverine Tual, 1,2,3 Mathilde Boulanger, 1,2 Marine Renier ${ }^{1,2}$ Romain Pons, ${ }^{5,6}$ Clément Piel, ${ }^{5,6}$ Camille Pouchieu, ${ }^{1,3}$ Yannick Lecluse, ${ }^{1,3}$ Stéphanie Perrier, ${ }^{1,3}$ Noémie Levêque-Morlais, GROUP AGRICAN-, ${ }^{3,4}$ AnneValérie Guizard, ${ }^{5,6,7}$ Alain Monnereau, ${ }^{5,6,8}$ Isabelle Baldi, 1,2,3Pierre Lebailly. 'Institut National de la Santé et de la Recherche Médicale (INSERM), Unité Mixte de Recherche (UMR) Interdisciplinaire pour la Prévention et le Traitement des Cancers 1086 ANTICIPE, Caen, France; ${ }^{2}$ University of Caen Normandie, Caen, France; ${ }^{3}$ Centre de Lutte contre le Cancer François Baclesse, Caen, France; ${ }^{4}$ Registre Général de Tumeurs du Calvados, Caen, France; ${ }^{5}$ EPICENE team, ISPED, Institut National de la Santé et de la Recherche Médicale (INSERM), Unité 1219, Bordeaux Population Health Research Center, Bordeaux, France; ${ }^{6}$ University of Bordeaux, Bordeaux, France; ${ }^{7}$ Hematological Malignancies Registry of Gironde, Beronie Institute, Comprehensive Cancer Center, Bordeaux, France; ${ }^{8}$ Centre Hospitalier Universitaire (CHU) de Bordeaux, Service de Médecine du Travail et Pathologies Professionnelles, Bordeaux, France
\end{abstract}

\subsection{6/OEM-2019-EPI.7}

Purpose Increased risks of Non-Hodgkin's Lymphomas (NHLs) have been reported among farmers in several metaanalyses, with exposure to pesticides as the main explanation. Few studies investigated associations by NHL subtypes and considering specific pesticides. From the French agricultural cohort AGRICAN, we assessed the role of benzimidazole 
fungicides use on NHL risk, overall and for multiple myeloma (MM), Chronic Lymphocytic Leukemia/Small Lymphocytic Lymphoma (CLL-SLL) and Diffuse Large B-cell Lymphoma (DLBCL).

Methods Nearly 182000 participants affiliated in a health assurance system reported in the enrollment questionnaire (2005-2007) lifetime pesticide use with start and end year on 13 crops and were followed until 2013. Using the crop-exposure matrix PESTIMAT, associations between NHLs and benzimidazole fungicides exposure (overall and for benomyl, carbendazim, fenzaflore, thiabendazole and thiophanate methyl) were estimated using Cox models.

Results After exclusion of prevalent cases, individuals with incomplete agricultural profession history data or a zero tracking period, 1,133 NHL incident cases were identified from cancer registries (269 MM, 244 CLL-SLL, 190 DLBCL). Nearly $20 \%$ of participants were considered exposed (median duration from 7 to 20 years according to active ingredient). Increased NHL risk was observed with exposure to benzimidazole, overall, on any crops (NHL: HR=1.13, 95\%CI $=0.94-$ 1.37, 150 cases, no duration relationship). Moreover, borderline positive associations were reported on specific crops: wheat/barley (NHL: HR=1.23, CLL-SLL: HR=1.42), beets (DLBCL, HR=2.19) and rape (DLBCL, 2.32). Significant increased risks were reported with $\mathrm{MM}$ for use of thiophanate-methyl on wheat/barley $(\mathrm{HR}=3.46,23$ exposed cases, no duration relationship) and with DLBCL for all 4 benzimidazole used on beets (HR from 2.34 to 2.57 and for the 2 used on rape $(\mathrm{HR}=2.39$ and 2.46$)$, although based on respectively 7 and 6 cases.

Conclusion These findings suggest positive associations between incidence of specific NHL subtypes, and exposure to benzimidazole as a chemical family or specific ingredients in this family.

\section{A.3 OCCUPATIONAL INSECTICIDE EXPOSURE, GLYCEMIC REGULATION AND BRONCHOCONSTRICTION: PRELIMINARY RESULTS FROM A SHORT-TERM COHORT STUDY AMONG SMALL-SCALE FARMERS IN UGANDA}

\footnotetext{
1,2Martin Rune Hassan Hansen*, 1,3Erik Jørs, ${ }^{1,4}$ Annelli Sandbæk, ${ }^{5}$ Daniel Sekabojja, ${ }^{6}$ John Ssempebwa, ${ }^{6}$ Ruth Mubeezi, ${ }^{7,8}$ Philipp Staudacher, ${ }^{9}$ Samuel Fuhrimann, 1,2 Vivi Schlünssen. ${ }^{1}$ Aarhus University, Aarhus C, Denmark; ${ }^{2}$ National Research Center for the Working Environment, Copenhagen, Denmark; ${ }^{3}$ Odense University Hospital, Odense, Denmark; ${ }^{4}$ Steno Diabetes Center Aarhus, Aarhus, Denmark; ${ }^{5}$ Uganda National Association of Community and Occupational Health, Kampala, Uganda; ${ }^{6}$ Makerere University, Kampala, Uganda; ' ${ }^{7}$ Eawag, Swiss Federal Institute of Aquatic Science and Technology, Dübendorf, Switzerland; ${ }^{8}$ Institute of Biogeochemistry and Pollutant Dynamics, ETH Zurich, Zürich, Switzerland; ${ }^{9}$ Utrecht Unversity, Utrecht, Netherlands
}

\subsection{6/OEM-2019-EPI.8}

Introduction Epidemiological studies have suggested associations between exposure to some classes of non-persistent insecticides, airways obstruction (Doust et al, 2014) and diabetes mellitus (Evangelou et al, 2016). Yet the evidence is limited by cross-sectional study designs and exposure metrics based on self-reported information only. The purpose of this study is to investigate these associations in a strong study design employing objective exposure metrics.

Methods From September 2018 to February 2019, we are conducting a short-term cohort study in a population of 370 small-scale farmers in Uganda, half of which are conventional farmers and the other half working towards organic certification. We examine participants before, during and after the October-November agricultural pesticide-spraying season. In each study round, participants perform spirometry, and glycemic regulation is assessed by analysis of glycated aemoglobin A (HbA1c) as well as fasting plasma glucose for a subgroup. Detailed questionnaire-based information on pesticide usage (frequency, intensity, duration, specific compounds, personal protective equipment etc.) is used to construct an internal task-exposure matrix. The task-exposure matrix will be validated by erythrocyte choline esterase (AchE) activity as a proxy for organophosphate and carbamate exposure, as well as passive pesticide samplers (silicone wristbands) worn by participants between the study rounds.

Results and discussion The baseline (pre-spraying season) data collection is finalized. Data collection for the two rounds of follow-up (during/after the spraying season) is ongoing. At the conference, we will present the first results on temporal changes across the spraying season in AchE and associations with glycated aemoglobin A, fasting plasma glucose and lung function.

\section{REFERENCES}

1. Doust, et al. Is pesticide exposure a cause of obstructive airways disease? European Respiratory Review 2014 June 1;23(132):180-92.

2. Evangelou, et al. Exposure to pesticides and diabetes: A systematic review and meta-analysis. Environment International 2016 May 1;91:60-8.

\section{A.4 ASSESSMENT OF PESTICIDE-RELATED POLLUTION AND OCCUPATIONAL HEALTH OF VEGETABLE FARMERS}

Jinky Leilanie Lu*. National Institutes of Health, University Of The Philippines Manila, Manila, Philippines

\subsection{6/OEM-2019-EPI.9}

Objectives This study assessed the pesticide exposure and the occupational health of the agricultural farmers in the Philippines. The study site is one of the largest vegetable-producing province in the Philippines.

Methods This study used both survey questionnaire and physical health assessment, including mental state examination. The pesticide exposure was estimated based on the duration of years of use of pesticide, as well as the amount applied per spray application. The data results were segregated between gender because women are also heavily engaged in agriculture in this part of the Philippines.

Results The results showed that pesticide exposure usually happened during agricultural activities such as spray application in the field $(63.9 \%)$, mixing (38.4\%), loading $(34.1 \%)$ and field re-entry $(9.7 \%)$ The most frequently used pesticides were Tamaron (OP), Selecron (OP), and Dithane (Carbamate). The mean years of pesticide use was 14.23 years for males, and 15.4 years for fe-males. For the exposure to pesticides, expressed in terms of number of years and amount used per spray application, the average exposure of the males was 2,024.43 ml-years; and for females, it was 993.55. Among farmers, 49\% complained of being sick because of work. Of those who got ill, a large percentage of this, $69.8 \%$, did not receive any medi-cal attention. The most prevalent health symptoms were muscle pain $(63.3 \%)$, easy fatigability $(52.4 \%)$, and loss of appetite (27.5\%). For the mini-mental state examination, abnormalities were found in $5.4 \%$ for males and $40.8 \%$ in females. The use of insecticide was associated with weak-ness, easy fatigability and weight loss. 